\title{
An Approximation to the Integral of the Circular Gaussian Distribution over an Offset Ellipse
}

\author{
By J. H. Cadwell
}

1. Introduction. We consider the evaluation of :

$$
P=\int_{-\infty}^{\infty} \int_{-\infty}^{\infty} \frac{1}{2 \pi} f(x, y) \exp \left\{-\frac{1}{2}\left(x^{2}+y^{2}\right)\right\} d x d y
$$

where $f(x, y)$ is unity inside the ellipse

$$
b^{2}(x-h)^{2}+a^{2}(y-k)^{2}=a^{2} b^{2},
$$

and zero elsewhere. The two special cases mentioned below have received a good deal of attention, comprehensive bibliographies will be found in [1] and [2]. If $a=b$, there is no loss of generality in taking $k=0$, and extensive tables exist for the offset circle integral. For the central ellipse, $h=k=0$, the integral can be deduced from that for the offset circle using a result given in [2]. An efficient method for the general case, based on Gaussian quadrature and the use of a table for Erf $(x)$, has been described in [3] and [4]. Tables for $P$ of limited extent will be found in [5] and [6], while an extensive table in inverse form is given in [4].

The purpose of this note is to describe an approximation, accurate to 3 decimal places, and suitable for an electronic computer. This does not employ a table of Erf $(x)$, and is thus more compact in terms of computer storage than the method of [3]. A comparison of computing times is given in Section 3.

The method is equivalent to a quadrature formula requiring the evaluation of the exponential at $3 a^{\prime}\left(a^{\prime}+1\right)+1$ points for an ellipse of semi-major axis $a$, where $a^{\prime}$ is the integral part of $(a+0.5)$. Increasing the number of evaluations by a factor of 4 reduces the maximum error to 0.0001 for semi-major axes less than 8 units. The average number of evaluations can be reduced considerably by neglecting terms where the index of the exponential falls below a prescribed value, since the number now depends on $h, k$ and tends to zero as they increase.

The basis of the method is the diffuse target concept, used in a number of weapon studies, e.g. in [7]. The function $f$ is approximated by

$$
f^{*}(x, y)=2 \exp -2\left\{\frac{b^{2}(x-h)^{2}+a^{2}(y-k)^{2}}{a^{2} b^{2}}\right\} \text {. }
$$

The surface $z=f(x, y)$ consists of a flat-topped elliptic cylinder of unit height, together with the region of the plane $z=0$ lying outside (2). The approximating surface is bell-shaped, and goes to infinity in all directions, hence the name "diffuse target." The value of

$$
\int_{-\infty}^{\infty} \int_{-\infty}^{\infty} x^{t}\left(f-f^{*}\right) d x d y
$$

Received February 11, 1963. Revised August 1, 1963. 
is zero for odd $t$ and $t=0$ and 2 . Similar results hold for moments about $0 x$. The expression

$$
P_{1}(a, b, h, k)=\frac{2 a b}{\left\{\left(a^{2}+4\right)\left(b^{2}+4\right)\right\}^{\frac{1}{3}}} \exp -2\left\{\frac{h^{2}}{a^{2}+4}+\frac{k^{2}}{b^{2}+4}\right\},
$$

obtained by replacing $f$ by $f^{*}$, is surprisingly accurate for small $a$ and $b$. If the semimajor axis does not exceed 0.5, errors are less than 0.0002 . Replacing $f$ by the approximation

$$
16\left\{\frac{b^{2}(x-h)^{2}+a^{2}(y-k)^{2}}{a^{2} b^{2}}\right\} \exp -4\left\{\frac{b^{2}(x-h)^{2}+a^{2}(y-k)^{2}}{a^{2} b^{2}}\right\},
$$

was found to lead to

$$
\begin{array}{r}
P_{2}(a, b, h, k)=\frac{16 a b}{\left\{\left(a^{2}+8\right)\left(b^{2}+8\right)\right\}^{\frac{1}{2}}}\left\{\frac{a^{2}\left(1+h^{2}\right)+8}{\left(a^{2}+8\right)^{2}}+\frac{b^{2}\left(1+k^{2}\right)+8}{\left(b^{2}+8\right)^{2}}\right\} \\
\times \exp -4\left\{\frac{h^{2}}{a^{2}+8}+\frac{k^{2}}{b^{2}+8}\right\} .
\end{array}
$$

This is of higher accuracy, and the combination $\left(4 P_{2}-P_{1}\right) / 3$, arrived at by matching moments up to order 4 , gives errors less than 0.0001 for semi-major axes less than 1. The method was also found to be useful in the analogous three dimensional problem of integrating a spherical Gaussian distribution over an offset ellipsoid. Below, a method of target diffusion based on a series of forms like (3) is considered. This method is able to deal with targets of any size. The use of more complicated expressions, typified by (6), can be extended and the results improved; but such extensions inevitably allow only a limited increase in target size. We seek a systematic method of diffusing an ellipse, involving the sum of a set of functions like (3). It turns out that a chain of 6 functions, with centres lying on an ellipse similar to the target, together with a central function, gives 3 decimal place accuracy for semi-major axes up to 1.5 units. By adding a chain of 12 centres lying outside this configuration, the semi-major axis can be extended to 2.5 units. Each such chain added extends the largest semi-major axis that can be dealt with accurately by one unit.

The target is thus regarded as split into a succession of elliptic annuli, each being diffused by its own chain of functions. There is, in addition, a small central ellipse. The number of such chains needed is the integral part of (semi-major axis +0.5 ). The maximum width of each annulus is equal to the full major axis of the central ellipse, and this width never exceeds one unit.

The disposition of diffusing points for an ellipse corresponds to the pattern for the circle from which it is derived by orthogonal projection. Thus the major axis alone enters in determining the number of diffusing functions. As a consequence, accuracy for a narrow ellipse is usually considerably higher than for a circle with the same major axis.

2. An Approximation for the Elliptic Annulus. We consider the use of 6 diffuse target functions, with centres lying on an ellipse with centre $(h, k)$ and semi-axes $(a d / \lambda, b d / \lambda)$, and equally spaced in eccentric angle. They lead to the approximat- 
ing function

$$
g \sum_{j=0}^{(6 r-1)} \exp -\frac{c}{2}\left\{\frac{(\lambda x-\lambda h-a d \cos \pi j / 3 r)^{2}}{a^{2}}+\frac{(\lambda y-\lambda k-b d \sin \pi j / 3 r)^{2}}{b^{2}}\right\} .
$$

The number $r$, inserted here for convenience, is at present taken to be 1 . The area lying between ellipses with centres at $(h, k)$ and with semi-axes $(a / \lambda, b / \lambda)$ and $(a, b)$ respectively is to be approximated by $(8)$, taking $\lambda$ greater than 1 . Matching moments up to order 2 leads to

$$
\left.\begin{array}{l}
c\left(\lambda^{2}-2 d^{2}+1\right)=4 \\
12 r g=c\left(\lambda^{2}-1\right)
\end{array}\right\}
$$

If in addition,

$$
6 d^{4}=\lambda^{4}+4 \lambda^{2}+1
$$

there will be agreement of moments up to order 4 . In order to cover semi-major axes up to 1.5 units, we assume that (5) is satisfactory up to 0.5 units and take $\lambda=3$. It is found that, while (10) gives $d=2.106$, a value of 2.094 gives better results over the range being considered.

This leads to the approximation

$$
\begin{aligned}
P_{1}(a / 3, b / 3, h, k) & +\frac{g a b}{\left\{\left(a^{2}+9 c\right)\left(b^{2}+9 c\right)\right\}^{\frac{1}{2}}} \\
& \times \sum_{j=0}^{5} \exp -\frac{c}{2}\left\{\frac{(3 h+a d \cos \pi j / 3)^{2}}{a^{2}+9 c}+\frac{(3 k+b d \sin \pi j / 3)^{2}}{b^{2}+9 c}\right\},
\end{aligned}
$$

for the integral, over an ellipse centre $(h, k)$ and with semi-axes $(a, b)$. The values of $g$ and $c$ follow from $(9)$, and for $\max (a, b)$ less than 1.5, errors are less than 0.00055 .

To extend the semi-major axis to 2.5 units, we can use the annulus between ellipses with semi-axes $(3 a / 5,3 b / 5)$ and $(a, b)$. Thus $\lambda=5 / 3$; and the values $d=1.3476$ and $r=2$, corresponding to a chain of 12 diffusion points, prove suitable. Thereafter successive values of $7 / 5,9 / 7 \cdots$ for $\lambda$ can be used, and for these later values, the value of $d$ is found from (10). An algorithm based on the use of successive annuli, each with width along the major axis less than or equal to unity, is described in the next section.

3. The Computational Procedure. We define the function

$$
\begin{aligned}
& B_{r}(\alpha, \beta, c, d, h, k)= \frac{\alpha \beta}{\left\{\left(\alpha^{2}+c\right)\left(\beta^{2}+c\right)\right\}^{\frac{1}{2}}} \\
& \quad \times \sum_{j=0}^{(6 r-1)} \exp -\frac{c}{2}\left\{\frac{(h+\alpha d \cos \pi j / 3 r)^{2}}{\alpha^{2}+c}+\frac{(k+\beta d \sin \pi j / 3 r)^{2}}{\beta^{2}+c}\right\} .
\end{aligned}
$$

We now determine $m$, the number of annuli, from

$$
m=\text { integer part (semi-major axis }+0.5 \text { ), }
$$

and define $\lambda_{r}, a_{r}$ and $b_{r}$ for $r$ from 1 to $m$ by 


$$
\lambda_{r}=(2 r+1) /(2 r-1), \quad a_{r}=a_{r+1} / \lambda_{r}, \quad b_{r}=b_{r+1} / \lambda_{r},
$$

with $a_{m+1}=a, b_{m+1}=b$. Next we construct $d_{r}, c_{r}$ and $g_{r}$ for $r$ from 1 to $m$, using the relations

$$
\begin{aligned}
& d_{1}=2.094, \quad d_{2}=1.3476, \quad 6 d_{r}^{4}=\lambda_{r}^{4}+4 \lambda_{r}{ }^{2}+1 \quad \text { for } \quad r>2, \\
& \left.\begin{array}{l}
c_{r}\left(\lambda_{r}^{2}-2 d_{r}^{2}+1\right)=4 \\
12 r g_{r}=c_{r}\left(\lambda_{r}^{2}-1\right)
\end{array}\right\} \text { for } r>0 .
\end{aligned}
$$

The approximation to the integral is given by

$$
P_{1}\left(a_{1}, b_{1}, h, k\right)+\sum_{r=1}^{m} g_{r} B_{r}\left(a_{r}, b_{r}, c_{r}, d_{r}, h, k\right) .
$$

It is easy to modify (12) slightly, so that a single subroutine covers the computation of both $P_{1}$ and the functions $B_{r}$.

In order to reduce the time involved in computing sines, cosines and exponentials, the following devices were employed. The program was written for any size of ellipse, but its use was in the main confined to semi-major axes less than 8 . The necessary sines and cosines for use up to $r=8$ were stored with the subroutine. An alternative method, requiring less storage, would be to build up the successive sines and cosines for a given $r$ from stored values of $\sin (\pi / 3 r)$ and $\cos (\pi / 3 r)$ by the addition formulae. Wherever the index of an exponential in (12) fell below -10 , the value of the corresponding term was taken to be zero.

Figures in Table 1 illustrate the economy effected by this device. Without its use the number of evaluations of the exponential function required is independent of $(h, k)$, and is given in the second column. When small terms are neglected the number needed depends on $(h, k)$, and the maximum requirement is given in the third column. For values of $\sqrt{h^{2}+k^{2}}$ exceeding $a$ the number will be appreciably smaller than this maximum. The last column gives the number of evaluations of exponential or error function required in the Gaussian integration of [4] for 3 decimal place accuracy. The method of the present note is faster for small ellipses, but slower for large ones. At higher levels of accuracy the method of [4] is more efficient, and the method described here is unsuitable for applications such as the preparation of the inverse tables of [4].

4. Tests of Accuracy. The approximation (16) was checked against the 4 decimal place values of [5]. These cover the region

$$
a, b=0.5(0.5) 3 \quad h, k=0(0.5) 3 .
$$

Six decimal place values were compared for the central ellipses

$$
a, b=0.5(0.5) 8 \quad h=k=0 .
$$

Values of the same accuracy were employed for the offset circle with

$$
a=b=0.5(0.5) 8 \quad \sqrt{\left(h^{2}+k^{2}\right)}=0(0.5) 10 .
$$

Since the approximation has a period of $\pi / 3$ in angle and is symmetrical in the line $\theta=\pi / 6$, the centres were taken along the $x$-axis, and along lines making angles $\pi / 12$ and $\pi / 6$ with it. 


\section{TABLE 1}

Number of evaluations of $\exp (x)$ and erf $(x)$

\begin{tabular}{c|c|c|c}
\hline Semi major axis & $\begin{array}{c}\text { Including all } \\
\text { terms }\end{array}$ & $\begin{array}{c}\text { Small terms } \\
\text { neglected }\end{array}$ & Method of ref. [4] \\
\hline 1 & 7 & 7 & 24 \\
2 & 19 & 19 & 24 or 32 \\
3 & 37 & 37 & 32 or 48 \\
4 & 61 & 61 & 32 or 48 \\
6 & 127 & 78 & 48 \\
8 & 217 & 91 & 48 or 64 \\
\hline
\end{tabular}

The values of $0.5,1.5 \cdots$ for the semi-major axis correspond to points of change in the number of annuli defined by (13). This was replaced by

$$
m=\text { integer part (semi-major axis }+0.499) \text {, }
$$

to ensure that these test cases corresponded to the most unfavourable conditions, where each annulus takes its maximum width of unity along the major axis.

In addition to the above tests, 500 values taken from the inverse tables [4] were also examined, to fill in regions not otherwise explored. The tables of [6] were also used as checks with semi-axes $(a, a / f)$ for $a, f=1,2,4$ and 8 . Values of $h, k$ are specified in these tables to cover probabilities down to at least 0.020 . Since these tables are to three decimal places, they only provide an upper limit to the errors of $(16)$.

The maximum error encountered in all tests was 0.00065 for a circle of radius 3.5. The figure below gives the error map for a circle of radius 2.5; an angular region of extent $\pi / 6$ suffices in view of the symmetry of the pattern. The loci are of positions of the origin relative to the circle that give equal errors of the approximation, the figures shown by each locus being of error multiplied by 10,000. The shaded area, extending to infinity, corresponds to errors less than 0.0001 . It will be seen that large errors occur at the centre, and just within and outside the circle. Errors at points of small probability are themselves small, and this behaviour is typical of results obtained in other cases.

If the values $d_{1}=2.098 d_{2}=1.347$ are used, more accurate results are obtained for the central ellipse, the maximum error being 0.0003 . For cases where a higher degree of accuracy is needed, and with a semi-major axis less than 1.5, we can add

$$
\tan ^{-1}\left(\frac{a h}{b k}\right)+\frac{\pi}{12},
$$

to the angle $\pi j / 3$ of (11). This, combined with the changed values of $d_{1}$ and $d_{2}$, reduces the maximum error of (11) to 0.0002 . However, the inclusion of these changes for $B_{1}$ in (16) slightly increases the maximum error recorded for all tests, giving a value of 0.0007 . The use of similar angular corrections in terms after $B_{1}$ were found not to lead to worthwhile improvements.

Tests in the offset circle case indicated that the errors for semi-major axes up to 12 are of much the same size as those described above. By replacing (17) by 


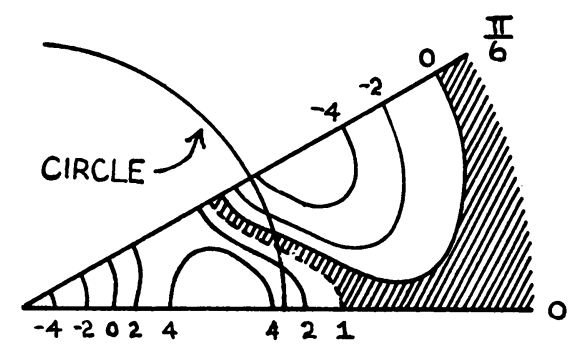

FIg. 1. Curves of equal error for a circle of radius 2.5 units

$$
m=\text { integer part (full major axis }+0.999 \text { ), }
$$

the number of exponentials to be evaluated is multiplied by 4 . Using $d_{1}=2.098$, $d_{2}=1.347$ and omitting (18), the resulting maximum error found was 0.0001 .

5. Some other applications. For the same integrand, taken over the interior of a simple closed curve $C$, the analogue of (3) leads to the approximation

$$
\begin{aligned}
\frac{A}{2 \pi\left\{\left(m_{20}+1\right)\left(m_{02}+1\right)-\right.} & \left.m_{11}^{2}\right\}^{\frac{1}{2}} \\
& \times \exp -\frac{\left(m_{02}+1\right) h^{2}-2 m_{11} h k+\left(m_{20}+1\right) k^{2}}{2\left\{\left(m_{20}+1\right)\left(m_{02}+1\right)-m_{11}^{2}\right\}} .
\end{aligned}
$$

Here, $A$ is the area and $(h, k)$ the mean centre of the region of integration, while

$$
A m_{i j}=\iint_{C}(x-h)^{i}(y-k)^{j} d x d y .
$$

This approximation will be useful for contours $C$ with a small enough maximum width. However, for the ellipse, the geometrical similarity of $C$ and of sections of the surface (3) indicates particularly favourable conditions for the method. Computations for a square of unit side gave a maximum error twice that for a circle of unit diameter.

If we consider the integrand

$$
\exp -\frac{1}{2}\left(x^{2}+y^{2}+z^{2}\right)
$$

taken over the interior of an ellipsoid with semi-axes $\left(a_{1}, a_{2}, a_{3}\right)$ and centre $\left(h_{1}\right.$, $h_{2}, h_{3}$ ), a diffusion function analogous to (3) gives the approximation

$$
I_{1}=\frac{10 \sqrt{5} a_{1} a_{2} a_{3}}{3\left\{2 \pi\left(a_{1}{ }^{2}+5\right)\left(a_{2}{ }^{2}+5\right)\left(a_{3}{ }^{2}+5\right)\right\}^{\frac{1}{2}}} \exp -\frac{5}{2}\left\{\sum_{i=1}^{3} \frac{h_{i}{ }^{2}}{\left(a_{i}{ }^{2}+5\right)}\right\} .
$$

The process corresponding to $(6)$ gives

$$
\begin{aligned}
& I_{2}=\frac{6250 a_{1} a_{2} a_{3}}{9\left\{2 \pi \left(3 a_{1}{ }^{2}+\right.\right.}\left.25)\left(3 a_{2}{ }^{2}+25\right)\left(3 a_{3}{ }^{2}+25\right)\right\}^{\frac{1}{2}} \\
& \quad \times\left\{\sum_{i=1}^{3} \frac{3 a_{i}{ }^{2}\left(1+h_{i}{ }^{2}\right)+25}{\left(3 a_{i}{ }^{2}+25\right)^{2}}\right\} \exp -\frac{25}{2}\left\{\sum_{j=1}^{3} \frac{h_{j}{ }^{2}}{\left(3 a_{j}{ }^{2}+25\right)}\right\} .
\end{aligned}
$$

The linear combination $\left(25 I_{2}-11 I_{1}\right) / 14$ is optimum for small axes. 
An examination of accuracy is difficult because of the lack of tabled values of the integral. However, if $a_{1}=a_{2}$ and $h_{1}=h_{2}=0$, conditions that include the offset sphere, an analytic solution is available. Computations show that, for this special case, errors of the linear combination of (23) and (24) for a semi-major axis less than one unit do not exceed 0.00003 . Experience in the 2 dimensional case suggests that, with the same limitation on major axis, accuracy of the same order can be expected in general. Similarly, it seems likely that the use of a set of diffusion points could be developed, as for the ellipse.

The author is indebted to the referee for suggesting the inclusion of Table 1, and for the data given in the last column of that table.

Mathematics Department,

Royal Aircraft Establishment,

Farnborough, Hants.,

England.

1. H. Weingarten \& A. R. DiDonato, "A table of generalized circular error," Math. Comp., v. 15, 1961, p. 169-173.

2. A. R. DiDonato \& M. P. Jarnagin, "A method for computing the circular coverage function," Math. Comp., v. 16, 1962, p. 347-355.

3. A. R. DiDonato \& M. P. JARnaGin, "Integration of the general bivariate Gaussian distribution over an offset circle," Math. Comp., v. 15, 1961, p. 375-382.

4. A. R. DiDonato \& M. P. JARnagin, Integration of the General Bivariate Gaussian Distribution Over an Offset Ellipse, NWL Report 1710, U. S. Naval Weapons Laboratory, Dahlgren, Virginia, 11 August 1960, unclassified.

5. H. H. GERMOND, Integration of the Gaussian Distribution Over an Offset Ellipse, RAND Corporation Report No. P-94, 28 July 1949, unclassified.

6. J. R. LowE, "A table of the integral of the bivariate normal distribution over an offset circle," J. R. Statist. Soc., B, v. 22, 1960, p. 177-187.

7. D. A: S. Fraser, "Generalized hit probabilities with a Gaussian target," Ann. Math. Statist., v. 22, 1951, p. 248-255. 